\title{
Manuscript
}

\section{Endohedral Hydrogen Bonding Templates the Formation of a Highly Strained Covalent Organic Cage Compound}

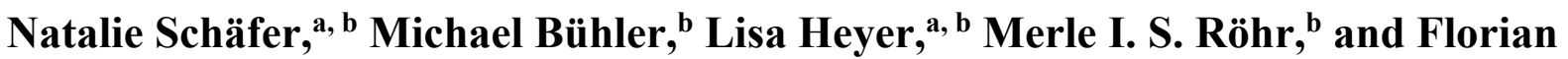 \\ Beuerle $^{* a, b}$
}

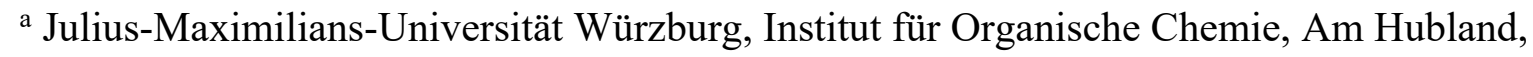 \\ 97074 Würzburg, Germany \\ b Julius-Maximilians-Universität Würzburg, Center for Nanosystems Chemistry (CNC), Theodor- \\ Boveri-Weg, 97074 Würzburg, Germany \\ *E-mail: florian.beuerle@uni-wuerzburg.de
}

\begin{abstract}
A highly strained covalent organic cage compound was synthesized from hexahydroxy tribenzotriquinacene (TBTQ) and a meta-terphenyl-based diboronic acid with an additional benzoic acid substituent in 2'-position. Usually, a $120^{\circ}$ bite angle in the unsubstituted ditopic linker favors the formation of a [4+6] cage assembly. Here we show that introduction of the benzoic acid group leads to a perfectly preorganized circular hydrogen-bonding array in the cavity of a trigonal-bipyramidal [2+3] cage, which energetically overcompensates the additional strain energy caused by the larger mismatch in bite angles for the smaller assembly. The strained cage compound was analyzed by mass spectrometry and ${ }^{1} \mathrm{H},{ }^{13} \mathrm{C}$ and DOSY NMR spectroscopy. DFT calculations revealed the energetic contribution of the hydrogen-bonding template to the cage stability. Furthermore, molecular dynamics simulations on early intermediates indicate an additional kinetic effect, as hydrogen-bonding also preorganizes and rigidifies small oligomers to facilitate the exclusive formation of smaller and more strained macrocycles and cages.
\end{abstract}

\section{Introduction}

Subcomponent self-assembly ${ }^{[1]}$ is a powerful tool for the one-pot-synthesis of complex nanoarchitectures directly from simple precursors. Depending on the interactions that connect the individual building blocks, supramolecular, ${ }^{[2]}$ metal-organic ${ }^{[3]}$ or dynamic covalent ${ }^{[4]}$ structures have been reported. For design purposes, geometrical concepts such as the directional bonding approach $^{[5]}$ allow control over geometry and topology of the obtained scaffolds, as these properties are directly encoded ${ }^{[6]}$ in the molecular structure of the building blocks. In recent years, a large variety of covalent organic cages ${ }^{[7]}$ with different size and shape ${ }^{[8]}$ have been designed and 
synthesized. Potential applications for these porous molecular materials ${ }^{[9]}$ range from molecular recognition, ${ }^{[10]}$ sensing, ${ }^{[11]}$ gas storage ${ }^{[8,}{ }^{82]}$ and separation, ${ }^{[13]}$ to encapsulation ${ }^{[14]}$ and reactivity control ${ }^{[15]}$ of large aromatic guests. In terms of complexity, rigid cages offer the tempting potential to hierarchically organize multiple functionalities with high spatial precision around the cage scaffold. Exohedral functionalization at the outer surface typically impacts the solubility ${ }^{[16]}$ or solidstate packing ${ }^{[8 f, 17]}$ of these modular porous units. Ultimately, the implementation of cross-linkable functions in the cage periphery can lead to covalently linked 'cage-to-framework' materials. ${ }^{[18]}$ On the other hand, endohedral functionalization of cages ${ }^{[19]}$ is less explored. Selected examples include the post-synthetic modification of a salicylbisimine cage via sixfold Williams ether synthesis ${ }^{[20]}$ or alkyne cages with inward-pointing pyridines. ${ }^{[21]}$ Besides chemical modulation of the pores, cavitydirecting functional sites might be utilized to template specific cage geometries or could interfere with each other during the assembly process. To date, template-assisted synthesis ${ }^{[22]}$ of covalent organic cage compounds has been rarely addressed. With proper choice of functional precursors however, switching between different cage geometries might be realized.

\section{Results and Discussion}

Here we report on the serendipitous formation of a highly strained [2+3] molecular cage $\mathbf{A}_{2} \mathbf{C}^{\mathbf{P h C O O H}_{3} \text { from hexahydroxy tribenzotriquinacene }}{ }^{[23]}$ (TBTQ) $\mathbf{A}^{[16]}$ and terphenyl diboronic acid derivative $\mathbf{C}^{\mathbf{P h C O O H}}$ through the formation of six boronate esters under water-removing conditions. DFT calculations suggest that the three endohedral $\mathrm{PhCOOH}$ groups in the trigonal-bipyramidal cage are perfectly preorganized for intramolecular hydrogen bonding, which overcompensates the additional strain energy in relation to larger [4+6] assemblies (Figure 1).

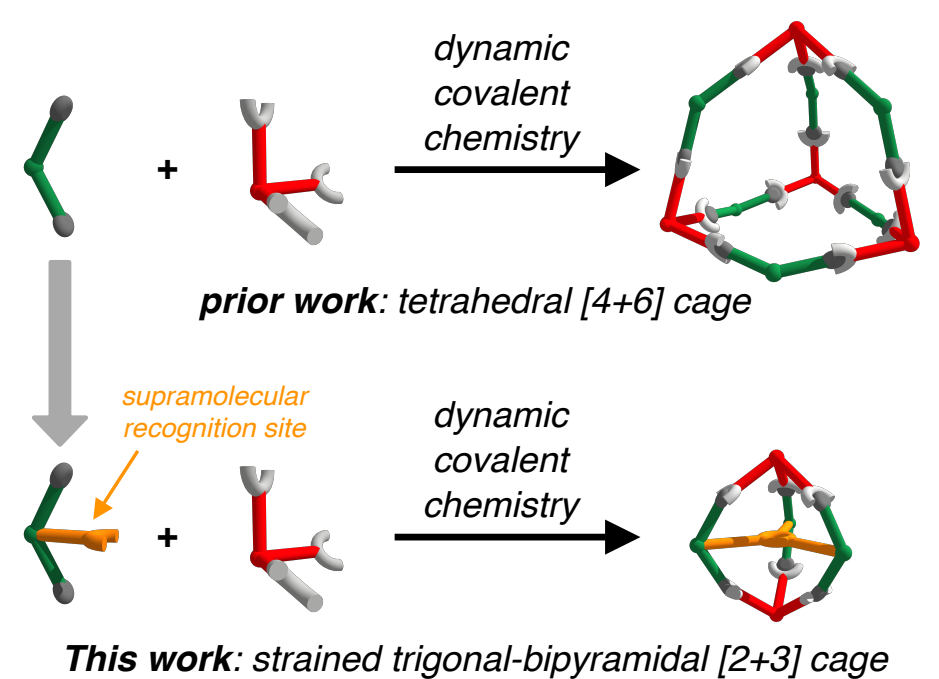

Figure 1. Supramolecular templation of strained covalent organic cages. 
In prior work, we reported on a series of shape-selective covalent organic cage compounds derived from orthogonal TBTQ A and diboronic acids with varying bite angles. ${ }^{[16,24]}$ For 2'-methyl[1,1':3',1' '-terphenyl]-4,4' '-diboronic acid ( $\left.\mathbf{C}^{\mathbf{M e}}\right)$ possessing a $120^{\circ}$ bite angle at the central phenylene unit, tetrahedral $\mathbf{A}_{\mathbf{4}} \mathbf{C}^{\mathbf{M e}} \mathbf{6}_{\mathbf{6}}$ cages were obtained as the only detectable self-assembly product. Since the six bent $\mathbf{C}^{\mathbf{M e}}$ linkers are octahedrally distributed around the cage pore, all Me groups in 2'-position are located within the cage cavities, whereas any substituent in 5'-position would point to the outside of the cages. Aiming for functionalized cavities, we envisioned the attachment of supramolecular binding sites, e.g., $\mathrm{COOH}$ groups within the cage cavities. Based on a procedure introduced by Höger and coworkers, ${ }^{[25]}$ we applied a modular synthetic approach (Scheme 1) that allows for easy modifications of linkers $\mathbf{C}$ at both 2'- and 5'-position by proper choice of aldehyde (1) and carboxylic acid (4) starting materials. Here, we synthesized a novel linker $\mathbf{C}^{\mathbf{P h C O O H}}$ possessing a $\mathrm{PhCOOH}$ substitutent in 2'-position as an endohedral recognition site and a ${ }^{t} \mathrm{BuPh}$ group in $5^{\prime}$-position to further enhance the solubility of the final assemblies. Diiodide $\mathbf{5}^{[25-26]}$ was synthesized in two literature-known steps and reacted to bispinacol ester $\mathbf{6}$ by twofold Miyaura borylation. Treatment of 6 with $\mathrm{BBr}_{3}$ followed by aqueous workup resulted in simultaneous cleavage of both pinacol and methyl ester protecting groups to give diboronic acid $\mathrm{C}^{\mathrm{PhCOOH}}$ in $76 \%$ yield.
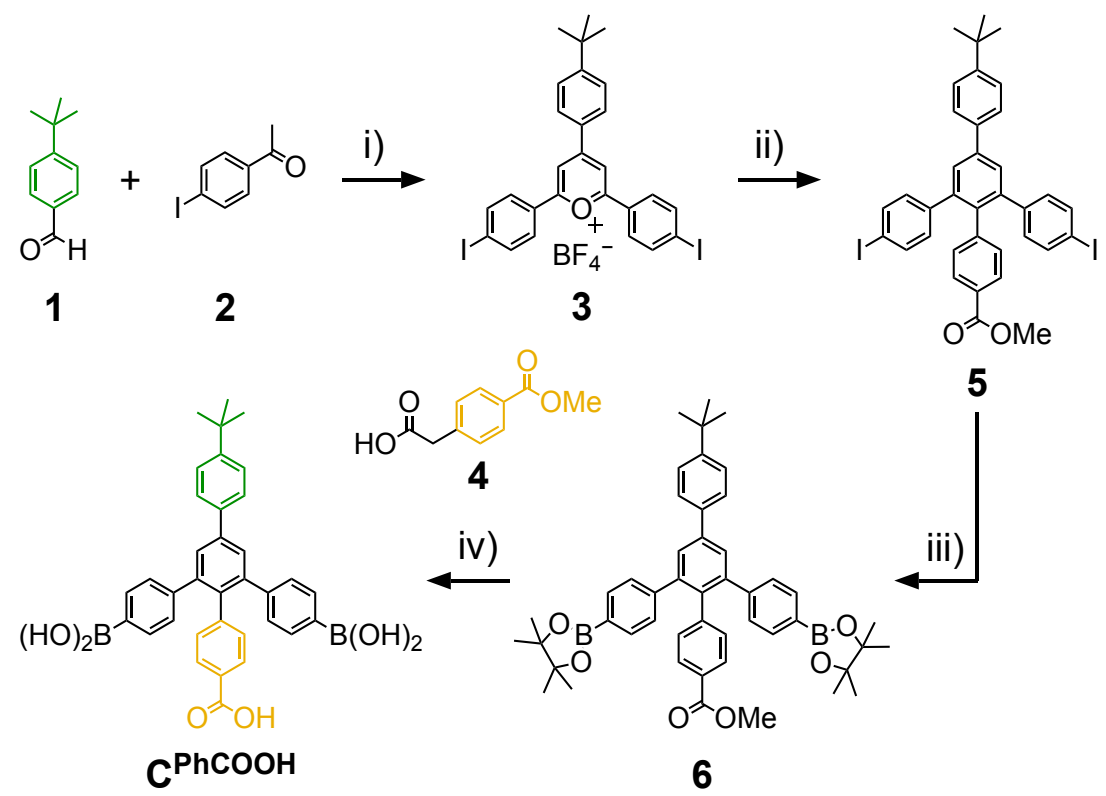

Scheme 1. Modular synthesis of diboronic acid $\mathbf{C}^{\mathrm{PhCOOH}}$ : i) $\mathrm{BF}_{3} \cdot \mathrm{OEt}_{2}, 100{ }^{\circ} \mathrm{C}, 2 \mathrm{~h}, 38 \%$; ${ }^{[25]}$ ii) $4^{[27]} /$ $\mathrm{NaOH}, \mathrm{MeOH}, 30 \mathrm{~min}$, then $\mathrm{Ac}_{2} \mathrm{O}, 160{ }^{\circ} \mathrm{C}, 2 \mathrm{~h}, 55 \%{ }^{; 26]}$ iii) $\mathrm{B}_{2} \mathrm{pin}_{2} / \mathrm{KOAc} / \mathrm{Pd}(\mathrm{dppf}) \mathrm{Cl}_{2}, \mathrm{DMF}$, reflux, 2.5h, $71 \%$, iv) $\mathrm{BBr}_{3}, \mathrm{CH}_{2} \mathrm{Cl}_{2}, 0{ }^{\circ} \mathrm{C} \rightarrow \mathrm{rt}, 3.75 \mathrm{~h}, 76 \%$.

For initial investigations regarding cage formation, we applied our established protocol ${ }^{[24]}$ and monitored the reaction of $\mathbf{A}$ and $\mathbf{C}^{\mathbf{P h C O O H}}$ in THF in the presence of $4 \AA$ molecular sieves by ${ }^{1} \mathrm{H}$ 
NMR spectroscopy. In contrast to $\mathbf{A}_{4} \mathbf{C}^{\mathbf{M e}}$, we did not observe the quantitative formation of a closed boronate ester assembly. Instead, rather broad signals and significant amounts of free catechols were observed even after prolonged reaction times and further addition of molecular sieves. MALDI-TOF mass spectrometry (MS) of the reaction mixture showed the two most prominent signals at $m / z=1883.24$ and 2382.70 (Figure S16), which correspond to smaller $\left[\mathbf{A}_{2} \mathbf{C}^{\mathbf{P h C O O H}_{2}+\mathrm{Na}}\right]^{+}$ and $\left[\mathbf{A}_{2} \mathbf{C}^{\mathbf{P h C O O H}_{3}+\mathrm{Na}}\right]^{+}$assemblies containing only two TBTQ units in a [2+3] cage or [2+2] macrocycle, respectively. This somewhat unexpected finding contradicts the directional bonding approach, ${ }^{[5]}$ which predicts a tetrahedral [4+6] assembly for the combination of a tritopic $90^{\circ}$ and a ditopic $120^{\circ}$ linker as it was realized for $\mathbf{A}_{4} \mathbf{C}^{\mathbf{M e}}{ }_{6 .}{ }^{[24]}$ For $\mathbf{C}^{\mathbf{P h C O O H}}$ however, a closer look at the structure of the molecular components and the MALDI-TOF MS data for the reaction mixture suggests a strong preorganization of two or three $\mathbf{C}^{\mathbf{P h C O O H}}$ molecules via hydrogen bonding between the $\mathrm{COOH}$ groups, which favors the formation of the strained [2+2] macrocycles and $[2+3]$ cage assemblies. In order to break these hydrogen bonds and to induce the formation of larger assemblies, e.g., [4+6] cages, up to two equivalents of acetic acid, referred to $\mathbf{C}^{\mathbf{P h C O O H}}$, were added to the reaction mixture. Monitoring the reaction progress via ${ }^{1} \mathrm{H}$ NMR (Figure S15) and MALDITOF MS gave identical data as in the previous attempts, thus indicating the strong driving force for supramolecular preorganization. However, no full conversion to the stoichiometric cage product was achieved, as usually observed for binary mixtures in 2:3 ratio. ${ }^{[16,24]}$ Both [2+2] macrocycles and [2+3] cages were simultaneously obtained as the two main products, accompanied by significant amounts of open oligomers. Presumably, additional strain is induced when closing the macrocyclic [2+2] intermediate with a third linker $\mathbf{C}^{\mathbf{P h C O O H}}$, thus shifting the equilibrium towards the macrocyclic fragment, which still possesses two unreacted catechol units. In order to facilitate full conversion and isolation of $\mathbf{A}_{2} \mathbf{C}^{\mathbf{P h C O O H}}{ }_{3}$, we screened for optimized reaction conditions. Finally, reaction of $\mathbf{A}$ and $\mathbf{C}^{\mathbf{P h C O O H}}$ in MeCN/THF 10:1 under reflux for 24 hours (Scheme 2) resulted in precipitation of a crude product. Resolvation of the isolated material in $\mathrm{MeOH}-\mathrm{d}_{4}$ induced complete disassembly into the monomeric building blocks. Based on ${ }^{1} \mathrm{H}$ NMR integration, the measured ratio of $\mathbf{A} / \mathbf{C}^{\mathbf{P h C O O H}}=2: 3$ indicates the predominant formation of a closed-shell [2+3] or [4+6] assembly rather than open [2+2] macrocycles or other oligomeric side products. MALDITOF MS for the isolated product (Figure 2c) revealed only one set of signals at $\mathrm{m} / \mathrm{z}=2323.29$ $\left[\mathbf{A}_{2} \mathbf{C}^{\mathbf{P h C O O H}_{3}-\mathrm{C}_{4} \mathrm{H}_{9}+\mathrm{Na}}\right]^{+}, \quad 2340.29 \quad\left[\mathbf{A}_{2} \mathbf{C}^{\mathbf{P h C O O H}_{3}-\mathrm{C}_{4} \mathrm{H}_{9}+\mathrm{K}}\right]^{+}, 2357.32 \quad\left[\mathbf{A}_{2} \mathbf{C}^{\mathbf{P h C O O H}_{3}}\right]^{+}, \quad 2364.27$ $\left[\mathbf{A}_{2} \mathbf{C}^{\mathbf{P h C O O H}_{3}+\mathrm{Li}}\right]^{+}$and $2381.30\left[\mathbf{A}_{\mathbf{2}} \mathbf{C}^{\mathbf{P h C O O H}}{ }_{3}+\mathrm{Na}\right]^{+}$that were all assigned to $\mathbf{A}_{\mathbf{2}} \mathbf{C}^{\mathbf{P h C O O H}}{ }_{3}$ and various monocationic adducts. Remarkably, the very high tendency for adduct formation with alkali metal ions indicates that the endohedral array of $\mathrm{COOH}$ groups might serve as an efficient binding station for metal ions. 

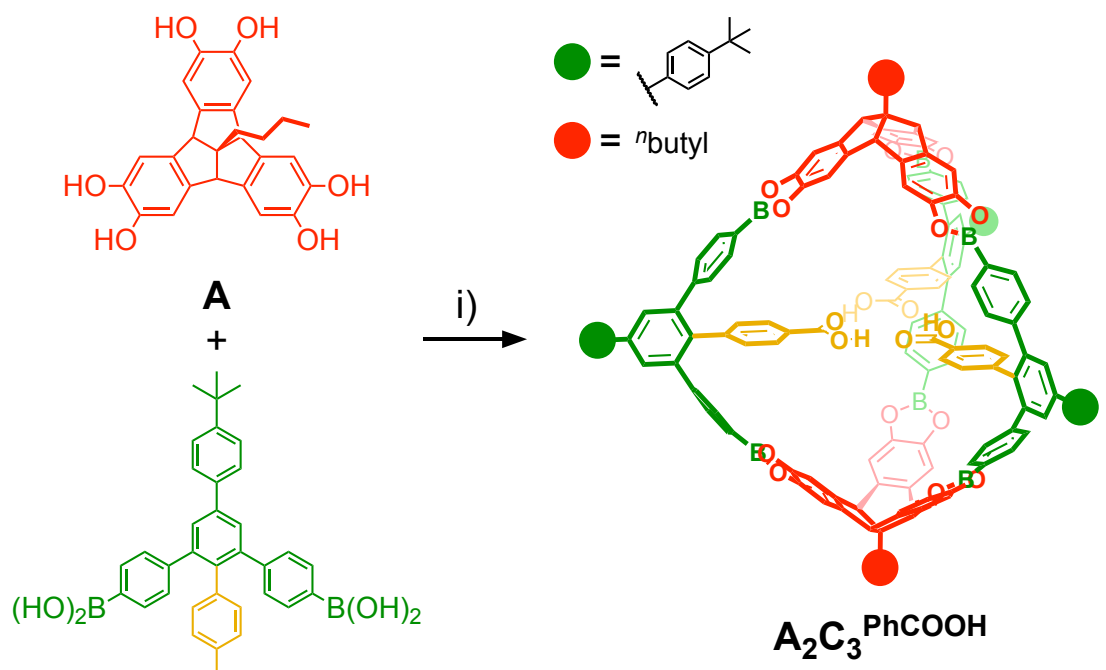

$\mathrm{C}^{\mathrm{PhCOOH}}$

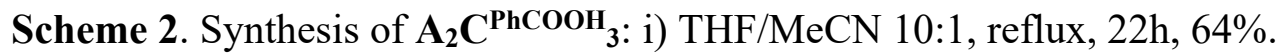

In contrast to the reaction in THF under equilibrating conditions (Figure S16), the absence of signals for other assemblies confirms the isolation of pure $\mathbf{A}_{2} \mathbf{C}^{\mathbf{P h C O O H}_{3}}$ cages from reaction in $\mathrm{MeCN}$. Presumably, closed-shell cages are significantly less soluble in $\mathrm{MeCN}$ as the more polar open intermediates, thus driving the dynamic formation of boronate esters towards completion. Finally, $\mathbf{A}_{2} \mathbf{C}^{\mathbf{P h C O O H}_{3}}$ cages are kinetically trapped by precipitation. After filtration and subsequent washing with $\mathrm{MeCN}$ and $n$-hexane, the raw product was further purified by suspending it in dry acetone for four hours at $60{ }^{\circ} \mathrm{C}$ to obtain cage $\mathbf{A}_{2} \mathbf{C}^{\mathbf{P h C O O H}}{ }_{3}$ in pure form. After work-up, the isolated cage was soluble in $\mathrm{CHCl}_{3}$ and the ${ }^{1} \mathrm{H}$ NMR spectrum (Figure 2a) shows only one single peak at $4.55 \mathrm{ppm}$ for the TBTQ bridgehead proton, thus further confirming the formation of one single cage species. Integration of protons corresponding to either TBTQ A or linker $\mathbf{C}^{\mathbf{P h C O O H}}$ confirmed the expected ratio of $\mathbf{A} / \mathbf{C}^{\mathbf{P h C O O H}}=2: 3$, whereas the occurrence of one single set of peaks for all chemically distinguishable protons argues for a highly symmetrical assembly.

In comparison to precursors 6 (in $\mathrm{CDCl}_{3}$ ) and $\mathbf{C}^{\mathrm{PhCOOH}}$ (in THF- $\mathrm{d}_{8}$ ), there is a striking upfield shift of roughly $0.5 \mathrm{ppm}$ for the aromatic protons of the $\mathrm{PhCOOH}$ substituents that are located in the cage cavity. We attribute this effect to additional shielding by the $\pi$-surface of the cage interior and, primarily, efficient hydrogen bonding between the closely arranged $\mathrm{PhCOOH}$ groups. For the acidic protons, a broad and barely detectable signal at $10.9 \mathrm{ppm}$ was observed. To estimate the cage size and ultimately differentiate between a [2+3] or [4+6] assembly, the solvodynamic diameter was determined via DOSY NMR measurements (Figure 2b). In $\mathrm{CDCl}_{3}$, a diffusion constant of $3.43 \times 10^{-10} \mathrm{~m}^{2} \mathrm{~s}^{-1}$ was obtained for the monodisperse cage species. According to the StokesEinstein equation, this value correlates to a solvodynamic diameter of $2.3 \mathrm{~nm}$, which is in very good agreement with a PM6 ${ }^{[28]}$-minimized space filling model (depicted as a semi-transparent sphere in 
Figure 2b). Cage $\mathbf{A}_{2} \mathbf{C}^{\mathbf{P h C O O H}_{3}}$ shows a considerably lower diameter than the previously reported tetrahedral cage $\mathbf{A}_{4} \mathbf{C}^{\mathbf{M e}}{ }_{6}(d=3.0 \mathrm{~nm}),{ }^{[24]}$ even though the protruding ${ }^{t} \mathrm{BuPh}$ substituents in 5'position at the bent linkers $\mathbf{C}^{\mathbf{P h C O O H}}$ are expected to result in even larger values for the tetrahedral $[4+6]$ assemblies, thus ultimately proving the formation of the smaller [2+3] cage.

a)
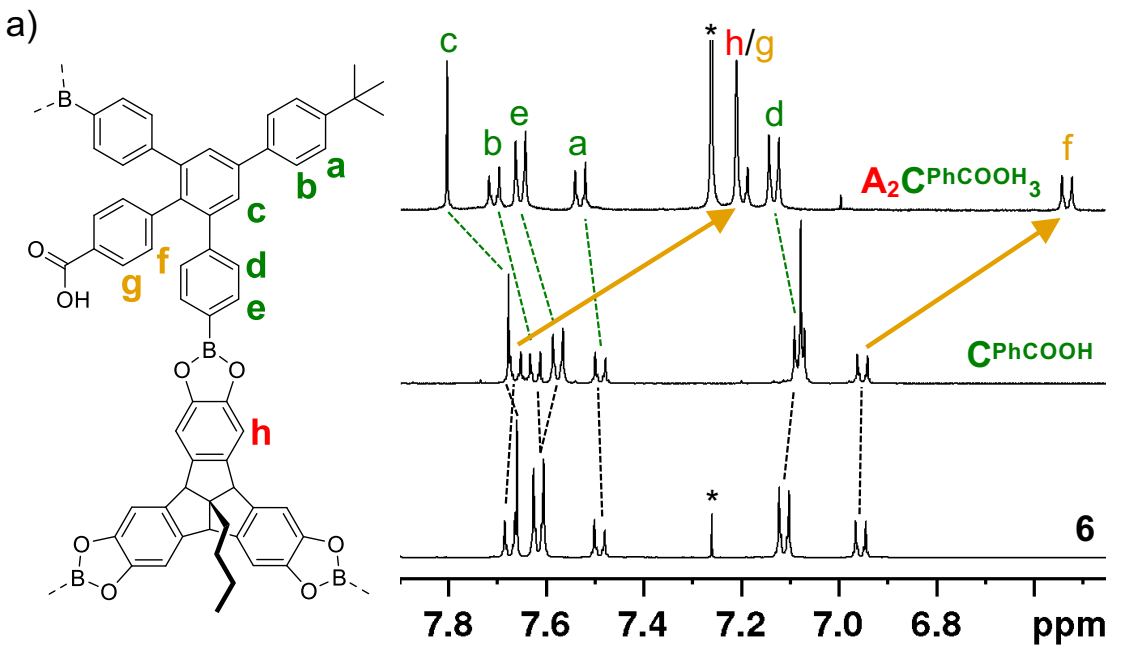

b)
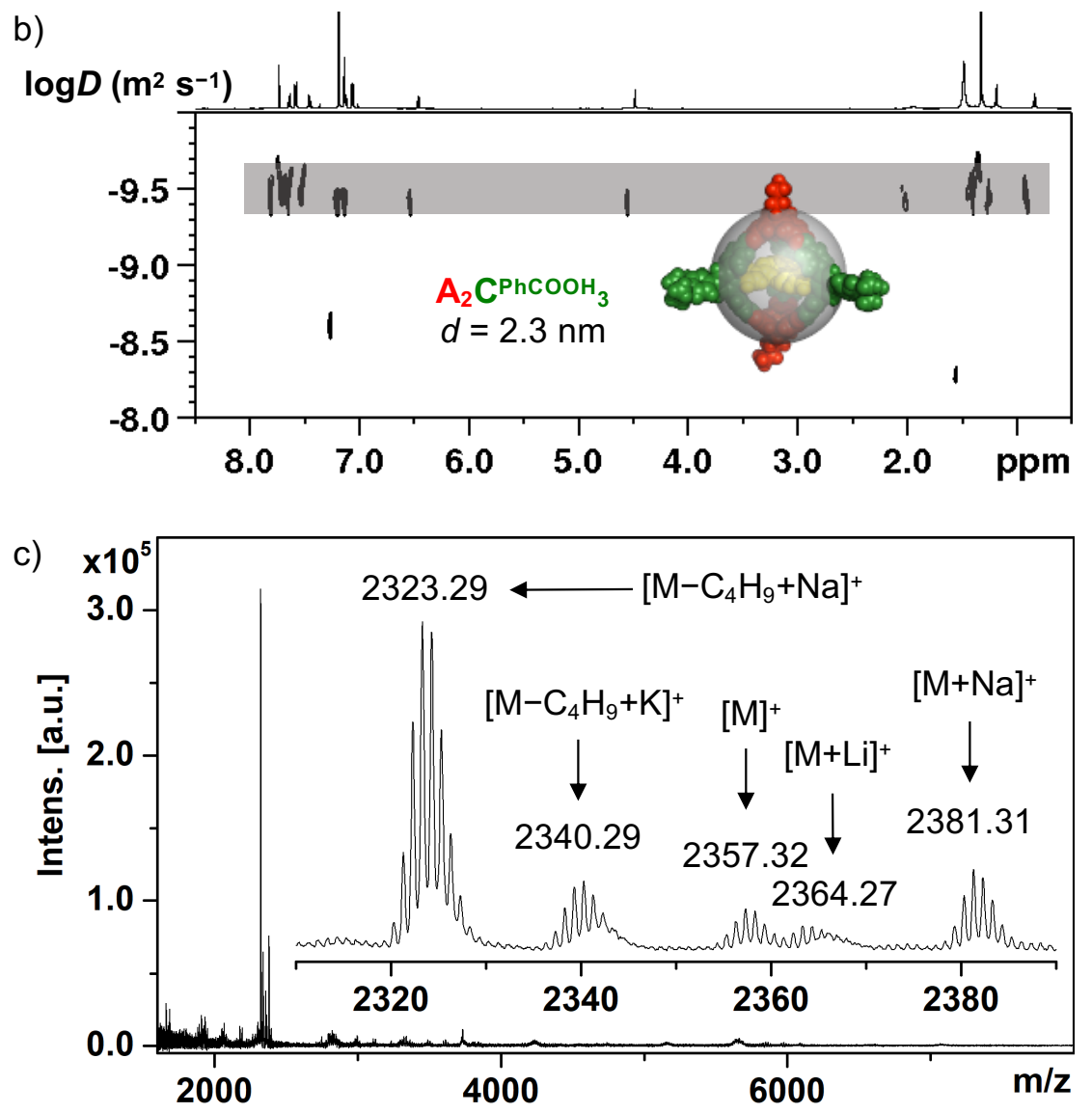

Figure 2. a) Aromatic region of ${ }^{1} \mathrm{H}$ NMR spectrum (400 MHz, rt) for $\mathbf{A}_{2} \mathbf{C}^{\mathbf{P h C O O H}_{3}}\left(\mathrm{CDCl}_{3}\right)$, $\mathbf{C}^{\text {PhCOOH }}\left(\mathrm{THF}-\mathrm{d}_{8}\right)$ and $\mathbf{6}\left(\mathrm{CDCl}_{3}\right)$, b) DOSY NMR (600 MHz, $\mathrm{CDCl}_{3}$, rt; inset shows PM6minimized space-filling model with the solvodynamic diameter indicated as a semi-transparent gray sphere) and c) MALDI-TOF MS for $\mathbf{A}_{2} \mathbf{C}^{\mathrm{PhCOOH}_{3} \text {. }}$ 
Remarkably, the simple exchange from $\mathrm{Me}$ to $\mathrm{PhCOOH}$ as endohedral substituent completely switched the cage size from a [4+6] to a [2+3] assembly and the geometrical arrangement of the linkers $\mathbf{C}$ from octahedral to trigonal planar, respectively. To evaluate if these selectivities are driven by different thermodynamic stabilities, we performed geometry optimizations employing DFT calculations with the $\omega \mathrm{B} 97 \mathrm{XD}^{[29]}$ functional and the def2-SVP ${ }^{[30]}$ basis set of cages in tetrahedral [4+6] and trigonal-bipyramidal [2+3] geometry for both $\mathbf{C}^{\mathbf{M e}}$ and $\mathbf{C}^{\mathbf{P h C O O H}}$. In order to decrease the computational demand, the apical ${ }^{n} \mathrm{Bu}$ substituents in $\mathbf{A}$ were replaced by Me groups and the exohedral ${ }^{t} \mathrm{BuPh}$ groups in 5 '-position for $\mathbf{C}^{\mathrm{PhCOOH}}$ were replaced by $\mathrm{H}$ atoms, as these groups are located on the outer cage surface and should not influence the inherent cage stability or preference for a specific topology. For $\mathbf{C}^{\mathbf{M e}}$, a formal transformation of one $\mathbf{A}_{4} \mathbf{B}^{\mathbf{M e}}{ }_{6}$ into two $\mathbf{A}_{2} \mathbf{B}^{\mathbf{M e}_{3}}$ cages revealed a difference in electronic energies of $+60 \mathrm{~kJ} \mathrm{~mol}^{-1}$ in the gas phase, indicating a significantly higher thermodynamic stability for the larger cage (Figure 3a). To adjust for any solvation effects, we simulated $\mathrm{MeCN}$ as solvent by applying a self-consistent field method using the integral equation formalism model (IEFPCM $)^{[31]}$ as implemented in the Gaussian $16^{[32]}$ quantum chemical software package. Again, an energy difference of $+62 \mathrm{~kJ} \mathrm{~mol}^{-1}$ was calculated, indicating that the equilibrium between the two cages is hardly affected by solvation but rather a consequence of the inherent stability of the individual cage geometries. In accordance with the directional bonding approach, this selectivity is matched in the synthetic experiment and is most presumably related to the inherent strain energy induced by the larger mismatch in the bite angles and a significant bending in the three struts for $\mathbf{C}^{\mathbf{M e}}$ (see Figure $3 \mathrm{a}$ ). For $\mathbf{C}^{\mathbf{P h C O O H}}$ however, DFT optimization in the gas phase revealed a completely reversed thermodynamic driving force, as the theoretical results indicate $\mathbf{A}_{2} \mathbf{C}^{\mathbf{P h C O O H}}{ }_{3}$ to be $-207 \mathrm{~kJ} \mathrm{~mol}^{-1}$ more stable in electronic energy than the larger [4+6] assembly (Figure 3a). For calculations with the MeCN continuum model, this energy difference is reduced to $-150 \mathrm{~kJ} \mathrm{~mol}^{-1}$, indicating the pronounced stabilization of polar $\mathrm{COOH}$ groups in polar aprotic solvents such as $\mathrm{MeCN}$. The geometry-optimized structures for both $\mathbf{A}_{4} \mathbf{C}_{6}$ cages show a very good overlap for the cage backbone (Figure S20a). As a response to the pore filling with the internal hydrogen bonding array of the $\mathrm{PhCOOH}$ groups, a slight deviation was observed for the $\mathbf{A}_{\mathbf{2}} \mathbf{C}_{\mathbf{3}}$ cages (Figure S20b). Therefore, we conclude that the inherent strain induced by the rigid scaffold of a specific cage stoichiometry does not depend on the inner substituents. However, attractive supramolecular interactions between the endohedral $\mathrm{PhCOOH}$ groups in the cage pores overcompensate the higher strain energy for the $\mathbf{A}_{2} \mathbf{C}^{\mathbf{P h C O O H}}{ }_{3}$ cage. 


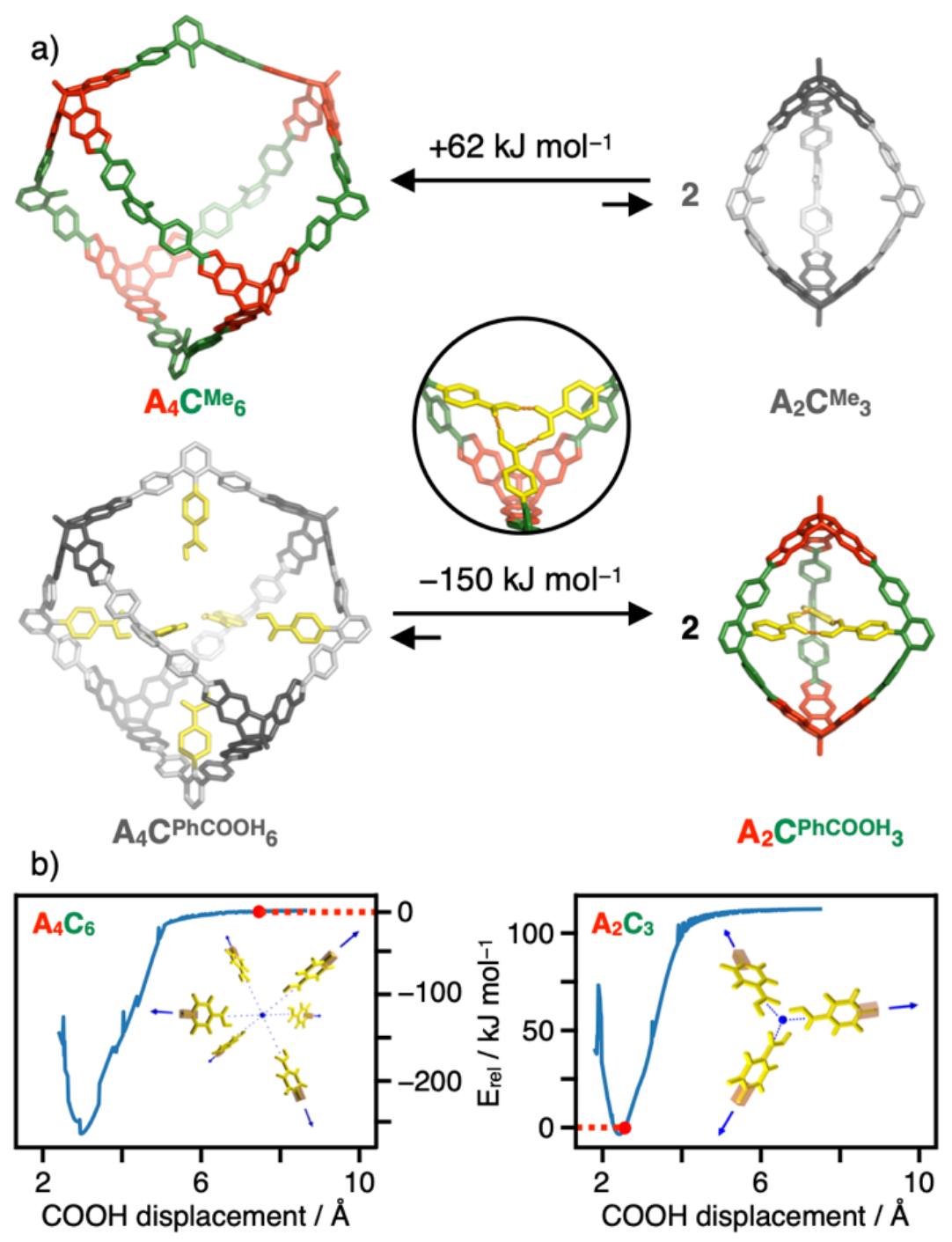

Figure 3. a) Equilibria between tetrahedral $\mathbf{A}_{\mathbf{4}} \mathbf{C}_{\mathbf{6}}$ and trigonal-bipyramidal $\mathbf{A}_{\mathbf{2}} \mathbf{C}_{\mathbf{3}}$ cages with endohedral $\mathrm{Me}$ (top) and $\mathrm{PhCOOH}$ (bottom) functionalization (electronic energies and geometryoptimized models derived from DFT calculations, $\mathrm{H}$ atoms are omitted for clarity); b) potential energy plots for the assembly of six or three $\mathrm{PhCOOH}$ moieties as realized in $\mathbf{A}_{4} \mathbf{C}^{\mathbf{P h C O O H}_{6}}$ and $\mathbf{A}_{2} \mathbf{C}^{\mathbf{P h C O O H}}{ }_{3}$, respectively (the actual distance in the DFT-optimized cages is indicated in red).

To further probe this stabilizing contribution, we performed relaxed scans for the endohedral substituents in both geometries (Figure 3b). For this purpose, we constructed model systems containing six and three benzoic acid molecules in the respective octahedral $\left(\mathbf{A}_{4} \mathbf{C}_{6}\right)$ or trigonalplanar $\left(\mathbf{A}_{2} \mathbf{C}_{3}\right)$ arrangement. To artificially adjust for varying cage sizes, we systematically scanned the distance of all $\mathrm{COOH}$ groups from the center of mass for both model systems. During these constrained optimizations, all benzoic acid molecules were kept fixed at the $\mathrm{C}-\mathrm{H}$ unit in 4-position (indicated as orange boxes in Figure $3 b$ ) to simulate the rigid character of the cage pores, while the molecules itself were allowed to structurally relax. The obtained potential energy profiles presented in Figure $3 \mathrm{~b}$ show that the actual arrangement of the three $\mathrm{PhCOOH}$ groups in $\mathbf{A}_{2} \mathbf{C}^{\mathbf{P h C O O H}}{ }_{3}$ is very 
close to the energetically most favorable distance, while the separation in the larger $\mathbf{A}_{\mathbf{4}} \mathbf{C}^{\mathbf{P h C O O H}} \mathbf{6}_{\mathbf{6}}$ is very far from the potential minimum. Essentially, there is no attractive interaction between the $\mathrm{COOH}$ groups at a distance of $7.49 \AA$ from the focal point in $\mathbf{A}_{4} \mathbf{C}^{\mathbf{P h C O O H}} \mathbf{6}$, whereas the $\mathbf{A}_{2} \mathbf{C}_{3}$ scaffold preorganizes the $\mathrm{COOH}$ groups almost perfectly for a circular threefold hydrogen-bonding motif (see inset in Figure 3a). To quantify this stabilizing interaction, we reoptimized the threefold array with DFT and the MeCN continuum model at both the distance realized in the cage and the most distant arrangement as reference. From the electronic energies, a binding energy of $-104 \mathrm{~kJ} \mathrm{~mol}^{-1}$ was calculated. Assuming that the inherent strain in $\mathbf{A}_{2} \mathbf{C}^{\mathbf{P h C O O H}} \mathbf{3}$ is the same as for the empty $\mathbf{A}_{2} \mathbf{C}^{\mathbf{M e}}$, a stabilizing contribution of $-106 \mathrm{~kJ} \mathrm{~mol}^{-1}$ per cage was estimated from the reaction equations in Figure 3a. The very good agreement between these two values further corroborate the subtle balance between the geometrical strain distributed in the cage backbone and any stabilizing interactions within the cage cavities.

Besides the hydrogen-bond mediated thermodynamic driving force for the formation of the more strained [2+3] cages, kinetic effects might also play a role, as hydrogen bonding could already fixate early intermediates during cage formation, thus directing any further reactions towards more strained smaller assemblies. As a key intermediate, we identified bisboronate ester $\mathbf{A C}^{\mathbf{P h C O O H}}{ }_{2}$, since hydrogen bonding between the $\mathrm{PhCOOH}$ moieties might severely restrict rotational motions in this molecule. To test this hypothesis, we performed molecular dynamics (MD) simulations for both $\mathbf{A} \mathbf{C}^{\mathbf{M e}}{ }_{2}$ and $\mathbf{A C}^{\mathbf{P h C O O H}_{2}}$ with the semi-empirical $\mathrm{PM}^{[28]}$ method along with the D3H4 ${ }^{[33]}$ correction for an adequate description of hydrogen bonding and dispersion as implemented in the MOPAC2016 ${ }^{[34]}$ program suite. As a measure for preorganization, we plotted the B-B distance of the two remaining unfunctionalized boronic acid moieties. For both structures, ten MD trajectories at $298 \mathrm{~K}$ with randomly generated starting configurations were calculated for $1 \mathrm{~ns}$ (see Figure 4 for

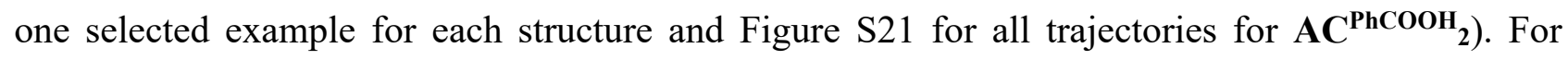
$\mathrm{AC}^{\mathrm{Me}}{ }_{2}$, the structure appeared to be rather flexible and a wriggling motion with a large variation of the B-B distance for the boronic acids ranging from 14 to $35 \AA$ was observed. For $\mathbf{A C}^{\mathbf{P h C O O H}_{2}}$ however, after a short time period of $100 \mathrm{ps,} \mathrm{most} \mathrm{trajectories} \mathrm{were} \mathrm{trapped} \mathrm{in} \mathrm{a} \mathrm{U-shaped} \mathrm{structure}$ with a fixed B-B distance of around $13 \AA$ (Figure 4a). Few simulations result in the population of an even more compact structure $\left(d_{\mathrm{B}-\mathrm{B}}=5.3 \AA\right)$, with one of the $\mathrm{PhCOOH}$ units forming a hydrogen bond to the opposite boronate ester (Figure S23). However, as this structure is approximately $18 \mathrm{~kJ} \mathrm{~mol}^{-1}$ higher in energy than the U-shaped arrangement, this metastable conformer is presumably only populated in neglectable amounts. For a more specific analysis, we used one representative starting configuration for $\mathbf{A C}^{\mathbf{P h C O O H}}{ }_{2}$ to calculate an ensemble of ten trajectories with randomly generated initial velocities based on a Maxwell-Boltzmann distribution at room 
temperature (Figure S22). Again, all simulations were quickly trapped in the two fixed geometries and the distance of the free boronic acids stayed constant for the remaining simulation. The average B-B distance for the prearranged U-shaped conformer of intermediate $\mathbf{A C}^{\mathbf{P h C O O H}}{ }_{2}$ is very close to the value of $9.6 \AA$ calculated for the DFT-optimized structures of the two isomers of the macrocycle $\mathbf{A}_{2} \mathbf{C}^{\mathbf{P h C O O H}}{ }_{2}$, which is assumed to act as the next intermediate within the mechanism of cage formation.
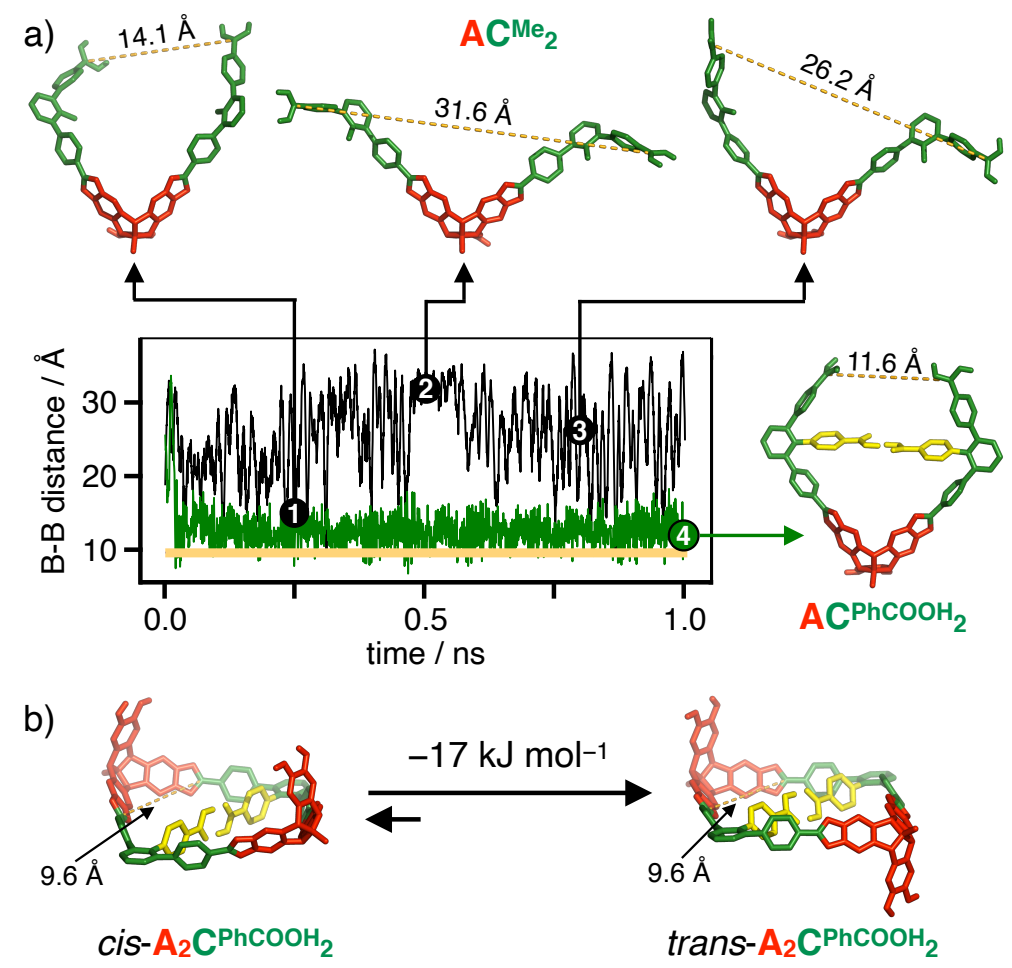

Figure 4. a) Molecular dynamics calculations of $\mathbf{A C}^{\mathrm{Me}}$ (black) and $\mathbf{A C}^{\mathrm{PhCOOH}_{2}}$ (green) for $1 \mathrm{~ns}$ at $298 \mathrm{~K}$ (1-3 display structures for $\mathbf{A} \mathbf{C}^{\mathbf{M e}}{ }_{2}$ at 250,500 and 800 ps, 4 displays structure for $\mathbf{A C}^{\mathbf{P h C O O H}} \mathbf{2}_{\mathbf{2}}$ at $1 \mathrm{~ns}$; yellow dashed lines indicate the B-B distance for the free boronic acids, the yellow line in

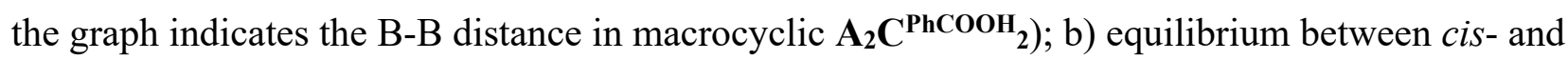

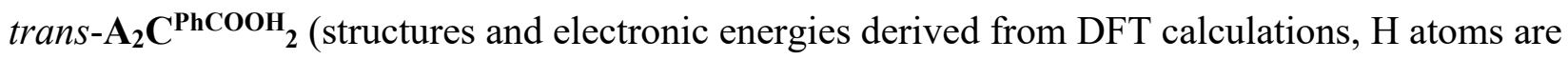
omitted for clarity).

Therefore, we postulate an additional kinetic effect that favors the preorganization of the building blocks towards the facile synthesis of the strained [2+2] macrocycle. Hereby, the formation of larger macrocycles, e.g. [3+3] or [4+4] is prevented, which are essential on-pathway intermediates in the synthesis of the larger tetrahedral [4+6] cages. Interestingly, DFT calculations showed that the trans-isomer is $-17 \mathrm{~kJ} \mathrm{~mol}^{-1}$ more stable than the cis-isomer (Figure $4 \mathrm{~b}$ ). Since the [2+3] cage can only be formed via the less preferred cis-isomer, the trans-macrocycle serves as a resting state, thus preventing further ring closure to the even more strained [2+3] cage assemblies. These subtle 
energy differences might be the explanation for the pronounced occurrence of the [2+2] macrocycles while performing the reactions under equilibrium conditions in THF (see Figures S1516). Dynamic covalent reaction of $\mathbf{A} / \mathbf{C}^{\mathbf{P h C O O H}}$ in a 1:1 ratio in THF further confirmed the inherent stability of the macrocyclic intermediates. MALDI-TOF MS of the reaction mixture predominantly showed signals at $m / z=1843.37$ and 3745.94, which can be assigned to [2+2] assemblies

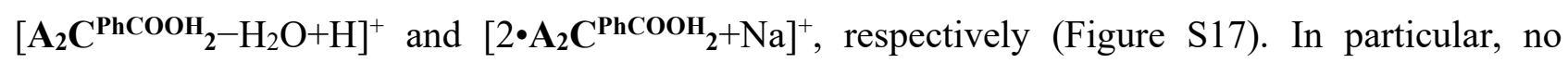
evidence for $\mathbf{A}_{2} \mathbf{C}^{\mathbf{P h C O O H}}{ }_{3}$ or other larger assemblies was found.

For the $\mathbf{A} / \mathbf{C}^{\mathbf{M e}}$ reaction mixture, $\mathbf{A}_{4} \mathbf{C}^{\mathbf{M e}}{ }_{6}$ is the only observable product despite a significant mismatch between optimal $\left(141^{\circ}\right)$ and actual $\left(120^{\circ}\right)$ bite angle for the linker $\mathbf{C}^{\mathrm{Me}}$. Therefore, strain energy is released in a social self-sorting experiment for a $\mathbf{A} / \mathbf{C}^{\mathbf{M e}} / \mathbf{D}$ mixture $\left(\mathrm{D}=2,5\right.$-di- ${ }^{n}$ butyl-1,4diboronic acid) by forming the mixed $\mathbf{A}_{4} \mathbf{C}^{\mathbf{M e}} \mathbf{4}_{\mathbf{2}}$ cage as the predominant product. ${ }^{[24,35]}$ To probe the effect of the endohedral $\mathrm{PhCOOH}$ groups on the formation of mixed cages, we performed a similar self-sorting experiment for a $\mathbf{A} / \mathbf{C}^{\mathbf{P h C O O H}} / \mathbf{D}$ by dissolving the building blocks in THF- $d_{8}$ in a 4:4:2 ratio, followed by the addition of $4 \AA$ molecular sieves as water removing agent. Progress of the reaction was monitored by ${ }^{1} \mathrm{H}$ NMR spectroscopy and MALDI-TOF MS. In contrast to $\mathbf{A} / \mathbf{C}^{\mathrm{Me}} / \mathbf{D}$, no formation of mixed cages was observed but rather narcissistic self-sorting into binary assemblies (Figure 5). MALDI-TOF MS of the reaction mixture confirms the concurrent formation of the cubic cage $\mathbf{A}_{\mathbf{8}} \mathbf{D}_{\mathbf{1 2}}{ }^{[16]}\left(\mathrm{m} / z=5953.83\right.$ for $\left.\left[\mathbf{A}_{\mathbf{8}} \mathbf{D}_{\mathbf{1 2}}+\mathrm{Na}\right]^{+}\right)$, macrocycle $\mathbf{A}_{\mathbf{2}} \mathbf{C}^{\mathbf{P h C O O H}} \mathbf{2}_{\mathbf{2}}$

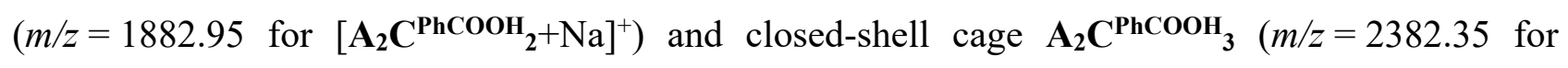
$\left[\mathbf{A}_{2} \mathbf{C}^{\mathbf{P h C O O H}_{3}+\mathrm{Na}}\right]^{+}$) with no evidence for any mixed assemblies. Apparently, molecular recognition of the $\mathrm{COOH}$ groups via the pronounced stabilization of $\mathbf{C}^{\mathbf{P h C O O H}}$-containing assemblies by intramolecular hydrogen bonding enforces narcissistic self-sorting of the linkers $\mathbf{C}^{\mathbf{P h C O O H}}$ and D into separated hydrogen-bonded structures and cubic cages, respectively. Formation of $\mathbf{A}_{\mathbf{8}} \mathbf{D}_{\mathbf{1 2}}$ was also observed in the ${ }^{1} \mathrm{H}$ NMR spectrum of the reaction mixture (Figure S18). Alongside, linker $\mathbf{C}^{\mathbf{P h C O O H}}$ was distributed between the closed $\mathbf{A}_{2} \mathbf{C}^{\mathbf{P h C O O H}_{3}}$ cage and open oligomers such as $\mathbf{A}_{2} \mathbf{C}^{\mathbf{P h C O O H}}{ }_{2}$ in a similar manner as for the $\mathbf{A} / \mathbf{C}^{\mathbf{P h C O O H}}$ mixture, thus indicating that the self-sorted system is under thermodynamic equilibrium. 
a)
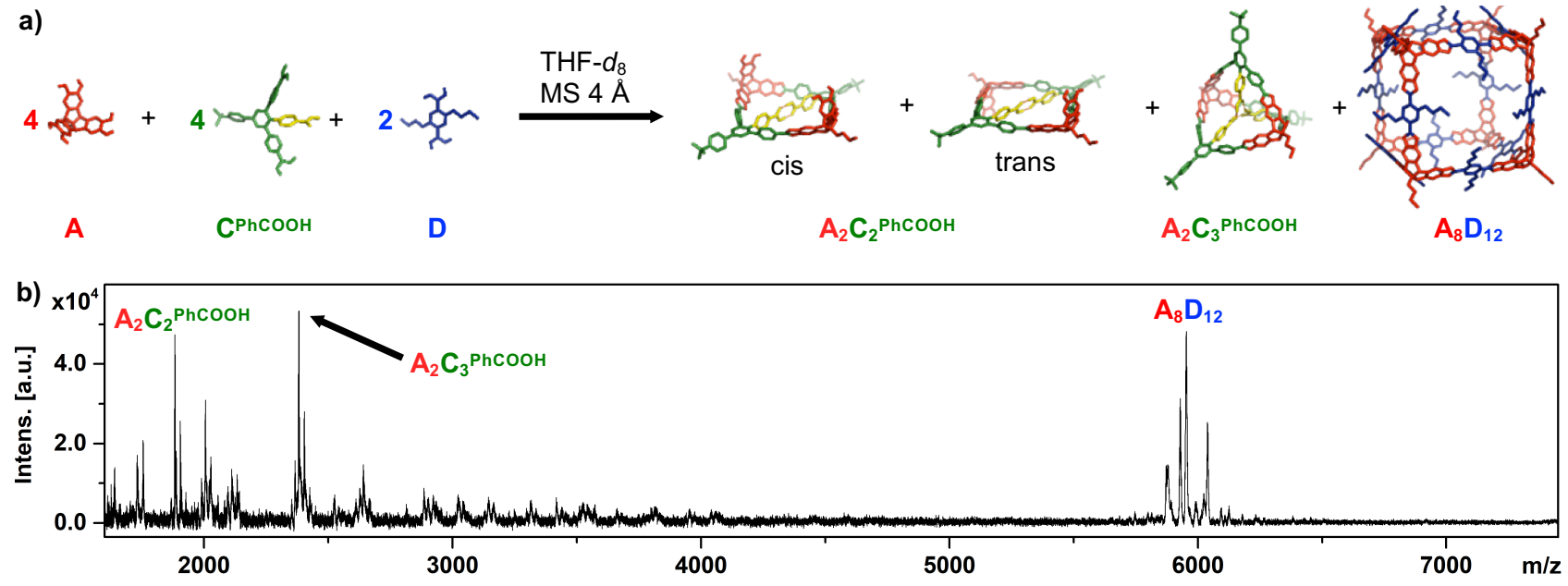

Figure 5. a) Molecular dynamics calculations of $\mathbf{A C}^{\mathbf{M e}}{ }_{2}$ (black) and $\mathbf{A C}^{\mathbf{P h C O O H}_{2}}$ (green) for $1 \mathrm{~ns}$ at $298 \mathrm{~K}$ (1-3 display structures for $\mathbf{A C}^{\mathrm{Me}}{ }_{2}$ at 250,500 and $800 \mathrm{ps}, 4$ displays structure for $\mathbf{A C}^{\mathbf{P h C O O H}} \mathbf{2}$ at $1 \mathrm{~ns}$; yellow dashed lines indicate the B-B distance for the free boronic acids, the

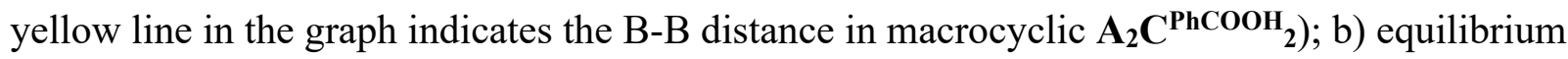

between cis- and trans- $\mathbf{A}_{2} \mathbf{C}^{\mathbf{P h C O O H}} \mathbf{2}_{\mathbf{2}}$ (structures and electronic energies derived from DFT calculations, $\mathrm{H}$ atoms are omitted for clarity).

\section{Conclusion}

A highly strained [2+3] trigonal-bipyramidal boronate ester cage has been synthesized by reacting hexahydroxy TBTQ A with $120^{\circ}$ diboronic acid $\mathbf{C}^{\mathbf{P h C O O H}}$ in 2:3 molar ratio in MeCN. As we have shown in previous work, the bite angle of $120^{\circ}$ for the ditopic linker $\mathbf{C}$ would usually trigger the assembly of tetrahedral [4+6] cages. For $\mathbf{C}^{\mathbf{P h C O O H}}$ however, pre-organization in dimers or trimers via intermolecular hydrogen-bonding between the $\mathrm{COOH}$ groups induces the formation of strained boronate ester bonds in smaller macrocycles and cages. MALDI-TOF MS and ${ }^{1} \mathrm{H}-,{ }^{13} \mathrm{C}$ - and DOSY NMR confirmed the formation of a highly symmetrical closed-shell assembly with the hydrodynamic radius being in good agreement with a PM6-minimized model for $\mathbf{A}_{2} \mathbf{C}^{\mathbf{P h C O O H}_{3} \text {. DFT }}$ calculations with a $\mathrm{MeCN}$ continuum model revealed that there is indeed a stabilizing contribution

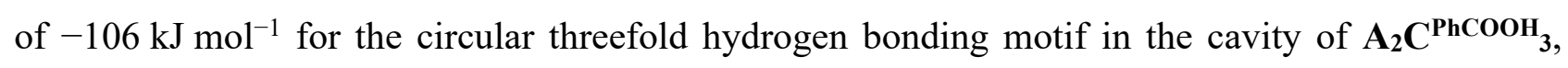
which overcompensates the implemented strain energy of $+62 \mathrm{~kJ} \mathrm{~mol}^{-1}$ when formally transforming one [4+6] into two [2+3] cages. This model system illustrates the limitations of the directional bonding approach for the prediction of cage geometry and topology through geometrical considerations for the molecular precursors. Supramolecular interactions between attached functional units and with solvent molecules can strongly influence the stability and formation pathways for complex dynamic covalent assemblies, thus giving access to structures that are normally not preferred. MD simulations on small oligomeric intermediates showed that there is also a kinetic contribution to the observed selectivity, since intramolecular hydrogen bonding in 
$\mathbf{A C}^{\mathbf{P h C O O H}}{ }_{2}$ already facilitates the formation of strained [2+2] macrocycles via fixation of

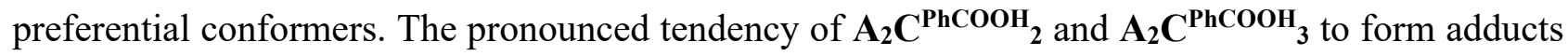
with monocations in MALDI-TOF MS experiments showcases the potential of these cages as ionic receptors. In future work, the implementation of switchable recognition sites within the cage pores could be utilized for a stimuli-responsive assembly of cages with different shapes and geometries.

\section{Experimental Section}

Chemicals and solvents were purchased from commercial suppliers Alfa Aesar, Merck, Acros, Abcr, Fischer and Sigma Aldrich and were used without further purification. Solvents were distilled prior to use. $\mathrm{CH}_{2} \mathrm{Cl}_{2}$ and DMF were dried with the solvent purification system "PureSolv MD 7" from Inert Technology. TLC sheets ALUGRAM Xtra SIL G/UV $\mathrm{UV}_{254}$ were purchased from Macherey-Nagel. Column chromatography was carried out with individually packed glass-columns of different sizes (silica, grain-size 40-63 $\mu \mathrm{m}$, Macherey-Nagel). NMR spectra were recorded on a Bruker avance III 400 or 600 spectrometer. Chemical shifts are indicated in ppm using the residual protonated solvent signal as internal standard $\left({ }^{1} \mathrm{H}\right.$ NMR: $7.26 \mathrm{ppm}$ for $\mathrm{CDCl}_{3}$ and $1.72 \mathrm{ppm}$ for THF- $d_{8} ;{ }^{13} \mathrm{C}$ NMR: $77.16 \mathrm{ppm}$ for $\mathrm{CDCl}_{3}$ and $67.21 \mathrm{ppm}$ for THF- $\left.d_{8}\right)$. Signal multiplicities are denoted as s (singlet), d (doublet), t (triplet), m (multiplet) and br (broad). Processing of the raw data was performed with the program Topspin 3.0. ${ }^{[36]}$ MALDI-TOF mass spectra were recorded on a ultrafleXtreme Bruker Daltonics (matrix: trans-2-(3-(4-t-Butylphenyl)-2-methyl-2propenylidene)-malononitrile, DCTB). ESI mass spectra were recorded on a micrOTOF focus spectrometer from Bruker Daltonics GmbH. Infrared spectra were taken on a Jasco FT/IR-430. Elemental analyses were performed on an Elementar CHNS 932 analyzer.

All geometry-optimizations for the cages, macrocycles and intermediates were performed with density functional theory (DFT), using the $\omega \mathrm{B} 97 \mathrm{XD}^{[29]}$ functional and the def2-SVP ${ }^{[30]}$ basis set. Additionally, a self-consistent reaction field method using the integral equation formalism model (IEFPCM) was used to simulate the solvent $\mathrm{MeCN}^{[31]}$ as implemented in the Gaussian 16 ${ }^{[32]}$ quantum chemical software package. Relaxed scans and molecular dynamics simulations were performed in the framework of the semi-empirical $\mathrm{PM}^{[28]}$ method along with the D3H4[33] correction for an adequate description of hydrogen bonds and dispersion by using the MOPAC2016 ${ }^{[34]}$ program suite. In order to decrease the computational demand, the ${ }^{t} \mathrm{BuPh}$ substituents in $\mathbf{C}$ were replaced by $\mathrm{H}$ atoms and the ${ }^{n} \mathrm{Bu}$ chains in TBTQ $\mathbf{A}$ were replaced by Me groups in all DFT and molecular dynamics calculations.

Compounds $\mathbf{3},{ }^{[25]} \mathbf{4},{ }^{[27]} \mathbf{5}^{[26]}$ and $\mathbf{A}^{[16]}$ have been synthesized according to previously published procedures. 
Bispinacol ester (6): Under nitrogen atmosphere, $\mathrm{B}_{2} \mathrm{pin}_{2}$ (1.19 g, $\left.4.68 \mathrm{mmol}, 2.5 \mathrm{eq}\right), \mathrm{Pd}(\mathrm{dppf}) \mathrm{Cl}_{2}$ (205 mg, $281 \mu \mathrm{mol}, 0.15 \mathrm{eq}), \mathrm{KOAc}$ (1.10 g, $11.2 \mathrm{mmol}, 6.0 \mathrm{eq})$ and diiodide 5 (1.40 g, $1.87 \mathrm{mmol}$, 1.0 eq) were dissolved in dry DMF $(120 \mathrm{~mL})$ and stirred at $90{ }^{\circ} \mathrm{C}$ for two hours and 30 minutes. Afterwards, DMF was removed under reduced pressure. The remaining solid was suspended in water $(170 \mathrm{~mL})$, treated in an ultrasonic bath for 40 minutes, filtrated and washed with water $(150 \mathrm{~mL})$ to remove the remaining DMF. Then, the solid was dissolved in EtOAc and filtrated over celite to remove the catalyst. The solvent was again removed under reduced pressure before the remaining solid was dissolved in a minimum amount of $\mathrm{CHCl}_{3}$ and impurities were precipitated by addition of $n$-hexane. The precipitate was filtered over a membrane filter and the solvent of the mother liquor was removed under reduced pressure. The remaining solid was dissolved in a minimum amount of EtOAc and the product was precipitated with $n$-hexane, filtrated and carefully washed by dropwise addition of cold EtOAc $(3 \mathrm{~mL})$ to obtain bispinacol ester 6 as a beige solid (1.00 g, 1.34 mmol, 71\%). m.p. $193.5{ }^{\circ} \mathrm{C}$; ${ }^{1} \mathbf{H}$ NMR (400 MHz, $\left.\mathrm{CDCl}_{3}, \mathrm{rt}\right): \delta=7.67$ (m, 4H, MeOOCPh- $\left.H / H^{4^{4} / 6}\right), 7.62\left(\mathrm{~m}, 6 \mathrm{H},{ }^{t} \mathrm{BuPh}-H / H^{3 / 5 / 3^{\prime \prime} / 5^{\prime \prime}}\right), 7.49\left(\mathrm{~d},{ }^{3} J_{\mathrm{HH}}=8.6 \mathrm{~Hz}, 2 \mathrm{H},{ }^{t} \mathrm{BuPh}-H\right), 7.11(\mathrm{~d}$, ${ }^{3} J_{\mathrm{HH}}=8.2 \mathrm{~Hz}, 4 \mathrm{H}, H^{2 / 6 / 2 " / 6}$ "), $6.95\left(\mathrm{~d},{ }^{3} \mathrm{~J}_{\mathrm{HH}}=8.6 \mathrm{~Hz}, 2 \mathrm{H}, \mathrm{MeOOCPh}-H\right), 3.87(\mathrm{~s}, 3 \mathrm{H}, \mathrm{COOCH}), 1.37$ (s, 9H, C(CH3) $\left.)_{3}\right), 1.34$ (s, 24H, BOC(CH3) $)$ ppm; $\left.{ }^{13} \mathbf{C ~ N M R ~ ( 1 0 1 ~ M H z , ~} \mathrm{CDCl}_{3}, \mathrm{rt}\right): \delta=167.23$, $150.91,144.54,144.51,142.30,140.73,137.43,136.74,134.35,131.82,129.40,128.85,128.56$, 127.78, 126.98, 126.01, 83.91, 52.07, 34.73, 31.49, 25.02 ppm; FT-IR (ATR): $\tilde{v}=660$ (s), 829 (m), 1087 (m), 1143 (m), 1276 (m), 1359 (s), 1608 (m), 1719 (m), 2977 (w) cm-1 MS (MALDI-TOF, DCTB in $\mathrm{CHCl}_{3}$, pos): $m / z=748.41[\mathrm{M}]^{+}, 771.40[\mathrm{M}+\mathrm{Na}]^{+}$; elemental analysis calcd (\%) for $\mathrm{C}_{48} \mathrm{H}_{54} \mathrm{~B}_{2} \mathrm{O}_{6}$ : C 77.02, H 7.27; found: C 76.52, H 7.43.

Diboronic acid $\mathbf{C}^{\mathrm{PhCOOH}}$ : Under nitrogen atmosphere, bispinacol ester 6 (800 mg, $1.07 \mathrm{mmol}$, 1.0 eq) was dissolved in dry $\mathrm{CH}_{2} \mathrm{Cl}_{2}(88 \mathrm{~mL})$ and cooled to $0{ }^{\circ} \mathrm{C} . \mathrm{BBr}_{3}(910 \mu \mathrm{L}, 2.41 \mathrm{~g}, 9.62 \mathrm{mmol}$, $9.0 \mathrm{eq}, d=2.65 \mathrm{~g} / \mathrm{mL}$ ) was added dropwise and the solution was stirred for one hour at $0{ }^{\circ} \mathrm{C}$, then two hours and 45 minutes at room temperature. Water $(100 \mathrm{~mL})$ was added to quench the reaction and the mixture was stirred overnight at room temperature. The mixture was extracted with EtOAc $(3 \times 50 \mathrm{~mL})$ and the combined organic phases were washed with water $(100 \mathrm{~mL})$ and brine and dried over $\mathrm{Na}_{2} \mathrm{SO}_{4}$. The solvent was removed under reduced pressure and the remaining solid was suspended in $\mathrm{CH}_{2} \mathrm{Cl}_{2}(150 \mathrm{~mL})$ and treated in an ultrasonic bath, before it was filtrated and washed with $\mathrm{CH}_{2} \mathrm{Cl}_{2}(40 \mathrm{~mL})$ and $n$-hexane $(40 \mathrm{~mL})$ to obtain $\mathbf{C}^{\mathbf{P h C O O H}}$ as a beige solid $(465 \mathrm{mg}, 815 \mu \mathrm{mol}$, 76\%). m.p. $222.1{ }^{\circ} \mathrm{C} ;{ }^{1} \mathbf{H}$ NMR (400 MHz, THF-ds, rt): $\delta=11.19$ (b, 1H, COOH), 7.68 (s, 2H, $\left.H^{4^{\prime} / 6^{\prime}}\right), 7.66\left(\mathrm{~d},{ }^{3} J_{\mathrm{HH}}=8.6 \mathrm{~Hz}, 2 \mathrm{H}, \mathrm{HOOCPh}-H\right), 7.62\left(\mathrm{~d},{ }^{3} J_{\mathrm{HH}}=8.6 \mathrm{~Hz}, 2 \mathrm{H},{ }^{t} \mathrm{BuPh}-H\right), 7.58(\mathrm{~d}$, ${ }^{3} J_{\mathrm{HH}}=8.2 \mathrm{~Hz}, 4 \mathrm{H}, H^{3 / 5 / 3}$ "'/5") $7.49\left(\mathrm{~d},{ }^{3} J_{\mathrm{HH}}=8.6 \mathrm{~Hz}, 2 \mathrm{H},{ }^{t} \mathrm{BuPh}-H\right), 7.08\left(\mathrm{~m}, 8 \mathrm{H}, H^{2 / 6 / 2}{ }^{\prime \prime / 6}\right.$ " $\left./ \mathrm{B}(\mathrm{OH})_{2}\right)$, 
$6.95\left(\mathrm{~d},{ }^{3} \mathrm{~J}_{\mathrm{HH}}=8.6 \mathrm{~Hz}, 2 \mathrm{H}, \mathrm{HOOCPh}-H\right), 1.35\left(\mathrm{~s}, 9 \mathrm{H}, \mathrm{C}\left(\mathrm{CH}_{3}\right)_{3}\right) ;{ }^{13} \mathbf{C}$ NMR $\left(101 \mathrm{MHz}, \mathrm{THF}-d_{8}, \mathrm{rt}\right)$ : $\delta=167.48,151.24,145.56,144.22,143.50,141.48,138.50,137.81,134.28,132.61,129.77,129.44$, 129.25, 128.89, 127.59, 126.51, 35.18, 31.68 ppm; FT-IR (ATR): $\tilde{v}=555$ (m), 657 (m), 704 (m), 826 (m), 1017 (m), 1335 (s), 1607 (m), 1694 (m), 2960 (w), 3393 (br) cm ${ }^{-1}$; MS (ESI-TOF, $\mathrm{MeOH} / \mathrm{MeCN}, \quad$ neg): $m / z=569.21 \quad[\mathrm{M}-\mathrm{H}]^{-} ; \quad$ elemental analysis calcd (\%) for $\mathrm{C}_{35} \mathrm{H}_{32} \mathrm{~B}_{2} \mathrm{O}_{6} \cdot 0.5 \mathrm{H}_{3} \mathrm{CCOOCH}_{2} \mathrm{CH}_{3}$ : C 72.34, H 5.91; found: C 72.16, H 5.93.

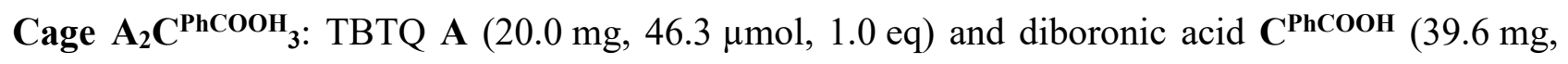
$69.4 \mu \mathrm{mol}, 1.5 \mathrm{eq})$ were dissolved in $\operatorname{THF}(0.5 \mathrm{~mL})$. MeCN $(5 \mathrm{~mL})$ was added and a violet precipitate formed. The suspension was stirred for 23 hours at $90{ }^{\circ} \mathrm{C}$. After cooling down to room temperature, the solid was filtrated and washed with $\mathrm{MeCN}(5 \mathrm{~mL})$ and $n$-hexane $(10 \mathrm{~mL})$. Then, the raw product was stirred in dry acetone $(9 \mathrm{~mL})$ for 4 hours at $60^{\circ} \mathrm{C}$ and the remaining solid was filtrated to obtain cage $\mathbf{A}_{2} \mathbf{C}^{\mathbf{P h C O O H}_{3}}$ as a light pink solid (34.8 mg, $14.7 \mu \mathrm{mol}, 64 \%$ ). ${ }^{1} \mathbf{H} \mathbf{~ N M R}$ (400 MHz, $\mathrm{CDCl}_{3}, \mathrm{rt}$ ): $\delta=10.92$ (s br, 3H, $\left.\mathrm{COOH}\right), 7.80$ (s, 6H, $\left.H^{4^{\prime} / 6^{\prime}}\right), 7.71\left(\mathrm{~d},{ }^{3} J_{\mathrm{HH}}=8.5 \mathrm{~Hz}, 6 \mathrm{H}\right.$, $\left.{ }^{t} \mathrm{BuPh}-H\right), 7.65\left(\mathrm{~d},{ }^{3} \mathrm{~J}_{\mathrm{HH}}=8.3 \mathrm{~Hz}, 12 \mathrm{H}, H^{3 / 5 / 3}{ }^{\prime \prime} / 5 "\right), 7.53\left(\mathrm{~d},{ }^{3} \mathrm{~J}_{\mathrm{HH}}=8.5 \mathrm{~Hz}, 6 \mathrm{H},{ }^{t} \mathrm{BuPh}-H\right), 7.20(\mathrm{~m}, 18 \mathrm{H}$, HOOCPh-H/TBTQ-Ar- $H), 7.13$ (d, ${ }^{3} J_{\mathrm{HH}}=8.3 \mathrm{~Hz}, 12 \mathrm{H}, H^{2 / 6 / 2 "}{ }^{\prime \prime}$ ) $) 6.53$ (d, ${ }^{3} J_{\mathrm{HH}}=8.5 \mathrm{~Hz}, 6 \mathrm{H}$, $\mathrm{HOOCPh}-H), 4.56$ (s, 6H, TBTQ-CH), 2.01 (m, 4H, $\left.\mathrm{CH}_{2} \mathrm{CH}_{2} \mathrm{CH}_{2} \mathrm{CH}_{3}\right), 1.38$ (m, 35H, $\left.\mathrm{C}\left(\mathrm{CH}_{3}\right)_{3} / \mathrm{CH}_{2} \mathrm{CH}_{2} \mathrm{CH}_{3}\right), 0.90\left(\mathrm{t},{ }^{3} \mathrm{~J}_{\mathrm{HH}}=7.1 \mathrm{~Hz}, 6 \mathrm{H}, \mathrm{CH}_{2} \mathrm{CH}_{3}\right) \mathrm{ppm} ;{ }^{13} \mathbf{C} \mathbf{N M R}\left(151 \mathrm{MHz}, \mathrm{CDCl}_{3}, \mathrm{rt}\right)$ : $\delta=169.30,151.01,148.42,145.16,145.07,142.25,140.26,140.02,139.73,137.56,134.04,130.77$, 129.92, 128.22, 127.09, 126.10, 125.71, 125.53, 124.20, 107.87, 67.21, 60.51, 40.06, 34.78, 31.52, 26.55, 23.55, 14.27 ppm; FT-IR (ATR): $\tilde{v}=553$ (s), 662 (s), 831 (m), 1017 (m), 1067 (m), 1328 (s), 1607 (m), 1689 (m), 2964 (w), 3384 (br) $\mathrm{cm}^{-1}$; MS (MALDI-TOF, DCTB in $\mathrm{CHCl}_{3}$, pos): $\mathrm{m} / z=$ 2323.29426 [M- $\left.\mathrm{C}_{4} \mathrm{H}_{9}+\mathrm{Na}\right]^{+}, 2340.28800\left[\mathrm{M}-\mathrm{C}_{4} \mathrm{H}_{9}+\mathrm{K}\right]^{+}, 2357.31661[\mathrm{M}]^{+}, 2381.47[\mathrm{M}+\mathrm{Na}]^{+}$.

\section{Conflicts of Interest}

There are no conflicts to declare.

\section{Acknowledgements}

Financial support from the DFG (BE 4808/2-1) is gratefully acknowledged. M. I. S. R. and M. B. wish to thank the Klaus Tschira Stiftung gGmbH (GSO/KT 21) for funding. 


\section{References}

[1] V. E. Campbell, J. R. Nitschke, Synlett 2008, 3077-3090.

[2] a) D. Beaudoin, F. Rominger, M. Mastalerz, Angew. Chem. Int. Ed. 2016, 55, 15599-15603; b) J. Luo, J.-W. Wang, J.-H. Zhang, S. Lai, D.-C. Zhong, CrystEngComm 2018, 20, 58845898.

[3] a) M. M. J. Smulders, I. A. Riddell, C. Browne, J. R. Nitschke, Chem. Soc. Rev. 2013, 42, 1728-1754; b) M. Han, D. M. Engelhard, G. H. Clever, Chem. Soc. Rev. 2014, 43, $1848-$ 1860; c) T. R. Cook, P. J. Stang, Chem. Rev. 2015, 115, 7001-7045.

[4] a) Y. Jin, C. Yu, R. J. Denman, W. Zhang, Chem. Soc. Rev. 2013, 42, 6634-6654; b) F. Beuerle, B. Gole, Angew. Chem. Int. Ed. 2018, 57, 4850-4878.

[5] P. J. Stang, B. Olenyuk, Acc. Chem. Res. 1997, 30, 502-518.

[6] J. M. Lehn, Chem. Eur. J. 2000, 6, 2097-2102.

[7] a) G. Zhang, M. Mastalerz, Chem. Soc. Rev. 2014, 43, 1934-1947; b) T. Hasell, A. I. Cooper, Nat. Rev. Mater. 2016, 1, 16053; c) M. Mastalerz, Acc. Chem. Res. 2018, 51, 2411-2422.

[8] a) X. Liu, Y. Liu, G. Li, R. Warmuth, Angew. Chem. Int. Ed. 2006, 45, 901-904; b) X. Liu, R. Warmuth, J. Am. Chem. Soc. 2006, 128, 14120-14127; c) Y. Liu, X. Liu, R. Warmuth, Chem. Eur. J. 2007, 13, 8953-8959; d) D. Xu, R. Warmuth, J. Am. Chem. Soc. 2008, 130, 75207521; e) M. Mastalerz, Chem. Commun. 2008, 4756-4758; f) T. Tozawa, J. T. A. Jones, S. I. Swamy, S. Jiang, D. J. Adams, S. Shakespeare, R. Clowes, D. Bradshaw, T. Hasell, S. Y. Chong, C. Tang, S. Thompson, J. Parker, A. Trewin, J. Bacsa, A. M. Z. Slawin, A. Steiner, A. I. Cooper, Nat. Mater. 2009, 8, 973-978; g) J. Sun, R. Warmuth, Chem. Commun. 2011, 47, 9351-9353; h) M. W. Schneider, I. M. Oppel, M. Mastalerz, Chem. Eur. J. 2012, 18, 41564160 ; i) G. Zhang, O. Presly, F. White, I. M. Oppel, M. Mastalerz, Angew. Chem. Int. Ed. 2014, 53, 1516-1520; j) S. M. Elbert, F. Rominger, M. Mastalerz, Chem. Eur. J. 2014, 20, 16707-16720; k) A. G. Slater, M. A. Little, A. Pulido, S. Y. Chong, D. Holden, L. Chen, C. Morgan, X. Wu, G. Cheng, R. Clowes, M. E. Briggs, T. Hasell, K. E. Jelfs, G. M. Day, A. I. Cooper, Nat. Chem. 2017, 9, 17-25; 1) J. C. Lauer, W. S. Zhang, F. Rominger, R. R. Schroder, M. Mastalerz, Chem. Eur. J. 2018, 24, 1816-1820.

[9] A. G. Slater, A. I. Cooper, Science 2015, 348, 988.

[10] a) Y. Ferrand, M. P. Crump, A. P. Davis, Science 2007, 318, 619-622; b) T. Mitra, K. E. Jelfs, M. Schmidtmann, A. Ahmed, S. Y. Chong, D. J. Adams, A. I. Cooper, Nat. Chem. 2013, 5, 276-281.

[11] a) M. Brutschy, M. W. Schneider, M. Mastalerz, S. R. Waldvogel, Adv. Mater. 2012, 24, 6049-6052; b) M. Brutschy, M. W. Schneider, M. Mastalerz, S. R. Waldvogel, Chem. Commun. 2013, 49, 8398-8400.

[12] a) M. Mastalerz, Chem. Eur. J. 2012, 18, 10082-10091; b) T. Kunde, E. Nieland, H. V. Schröder, C. A. Schalley, B. M. Schmidt, Chem. Commun. 2020, 56, 4761-4764. 
[13] M. Liu, L. Zhang, M. A. Little, V. Kapil, M. Ceriotti, S. Yang, L. Ding, D. L. Holden, R. Balderas-Xicohténcatl, D. He, R. Clowes, S. Y. Chong, G. Schütz, L. Chen, M. Hirscher, A. I. Cooper, Science 2019, 366, 613-620.

[14] a) C. Zhang, Q. Wang, H. Long, W. Zhang, J. Am. Chem. Soc. 2011, 133, 20995-21001; b) C. Yu, Y. Jin, W. Zhang, Chem. Rec. 2015, 15, 97-106; c) M. Ortiz, S. Cho, J. Niklas, S. Kim, O. G. Poluektov, W. Zhang, G. Rumbles, J. Park, J. Am. Chem. Soc. 2017, 139, 42864289.

[15] V. Leonhardt, S. Fimmel, A.-M. Krause, F. Beuerle, Chem. Sci. 2020, 11, 8409-8415.

[16] S. Klotzbach, T. Scherpf, F. Beuerle, Chem. Commun. 2014, 50, 12454-12457.

[17] a) M. J. Bojdys, M. E. Briggs, J. T. A. Jones, D. J. Adams, S. Y. Chong, M. Schmidtmann, A. I. Cooper, J. Am. Chem. Soc. 2011, 133, 16566-16571; b) M. W. Schneider, I. M. Oppel, H. Ott, L. G. Lechner, H.-J. S. Hauswald, R. Stoll, M. Mastalerz, Chem. Eur. J. 2012, 18, 836847.

[18] a) Y. Kim, J. Koo, I.-C. Hwang, R. D. Mukhopadhyay, S. Hong, J. Yoo, A. A. Dar, I. Kim, D. Moon, T. J. Shin, Y. H. Ko, K. Kim, J. Am. Chem. Soc. 2018, 140, 14547-14551; b) Q. Zhu, X. Wang, R. Clowes, P. Cui, L. Chen, M. A. Little, A. I. Cooper, J. Am. Chem. Soc. 2020, $142,16842-16848$.

[19] a) J. Yang, B. Chatelet, D. Hérault, J.-P. Dutasta, A. Martinez, Eur. J. Org. Chem. 2018, 5618-5628; b) J. Yang, B. Chatelet, V. Dufaud, D. Hérault, S. Michaud-Chevallier, V. Robert, J.-P. Dutasta, A. Martinez, Angew. Chem. Int. Ed. 2018, 57, 14212-14215.

[20] M. W. Schneider, I. M. Oppel, A. Griffin, M. Mastalerz, Angew. Chem. Int. Ed. 2013, 52, $3611-3615$.

[21] A. Burgun, P. Valente, J. D. Evans, D. M. Huang, C. J. Sumby, C. J. Doonan, Chem. Commun. 2016, 52, 8850-8853.

[22] a) A. R. Stefankiewicz, M. R. Sambrook, J. K. M. Sanders, Chem. Sci. 2012, 3, 2326-2329;

b) L. Y. Sun, N. Sinha, T. Yan, Y. S. Wang, T. T. Y. Tan, L. Yu, Y. F. Han, F. E. Hahn, Angew. Chem. Int. Ed. 2018, 130, 5161-5165.

[23] a) D. Kuck, Chem. Rev. 2006, 106, 4885-4925; b) D. Kuck, Chem. Rec. 2015, 15, 10751109; c) A. Dhara, F. Beuerle, Synthesis 2018, 50, 2867-2877.

[24] S. Klotzbach, F. Beuerle, Angew. Chem. Int. Ed. 2015, 54, 10356-10360.

[25] S. Klyatskaya, N. Dingenouts, C. Rosenauer, B. Müller, S. Höger, J. Am. Chem. Soc. 2006, $128,3150-3151$.

[26] A. B. Vliegenthart, F. A. L. Welling, M. Roemelt, R. J. M. Klein Gebbink, M. Otte, Org. Lett. 2015, 17, 4172-4175.

[27] R. Breslow, P. Marks, A. Mahendran, Y. Yao (Columbia University), US20180118709, 2018.

[28] J. J. P. Stewart, J. Mol. Model. 2007, 13, 1173-1213.

[29] J.-D. Chai, M. Head-Gordon, Phys. Chem. Chem. Phys. 2008, 10, 6615-6620. 
[30] F. Weigend, R. Ahlrichs, Phys. Chem. Chem. Phys. 2005, 7, 3297-3305.

[31] J. Tomasi, B. Mennucci, R. Cammi, Chem. Rev. 2005, 105, 2999-3093.

[32] M. J. Frisch, G. W. Trucks, H. B. Schlegel, G. E. Scuseria, M. A. Robb, J. R. Cheeseman, G. Scalmani, V. Barone, G. A. Petersson, H. Nakatsuji, X. Li, M. Caricato, A. V. Marenich, J. Bloino, B. G. Janesko, R. Gomperts, B. Mennucci, H. P. Hratchian, J. V. Ortiz, A. F. Izmaylov, J. L. Sonnenberg, Williams, F. Ding, F. Lipparini, F. Egidi, J. Goings, B. Peng, A. Petrone, T. Henderson, D. Ranasinghe, V. G. Zakrzewski, J. Gao, N. Rega, G. Zheng, W. Liang, M. Hada, M. Ehara, K. Toyota, R. Fukuda, J. Hasegawa, M. Ishida, T. Nakajima, Y. Honda, O. Kitao, H. Nakai, T. Vreven, K. Throssell, J. A. Montgomery Jr., J. E. Peralta, F. Ogliaro, M. J. Bearpark, J. J. Heyd, E. N. Brothers, K. N. Kudin, V. N. Staroverov, T. A. Keith, R. Kobayashi, J. Normand, K. Raghavachari, A. P. Rendell, J. C. Burant, S. S. Iyengar, J. Tomasi, M. Cossi, J. M. Millam, M. Klene, C. Adamo, R. Cammi, J. W. Ochterski, R. L. Martin, K. Morokuma, O. Farkas, J. B. Foresman, D. J. Fox, Gaussian 16 Rev. B.01, Gaussian, Inc., Wallingford, CT (USA), 2016.

[33] a) S. Grimme, J. Antony, S. Ehrlich, H. Krieg, J. Chem. Phys. 2010, 132, 154104; b) J. Řezáč, P. Hobza, J. Chem. Theory Comput. 2012, 8, 141-151.

[34] J. J. P. Stewart, MOPAC2016, Stewart Computational Chemistry, Colorado Springs, CO (USA), http://OpenMOPAC.net, 2016.

[35] F. Beuerle, S. Klotzbach, A. Dhara, Synlett 2016, 27, 1133-1138.

[36] TopSpin 3.0, Bruker, www.bruker.com. 